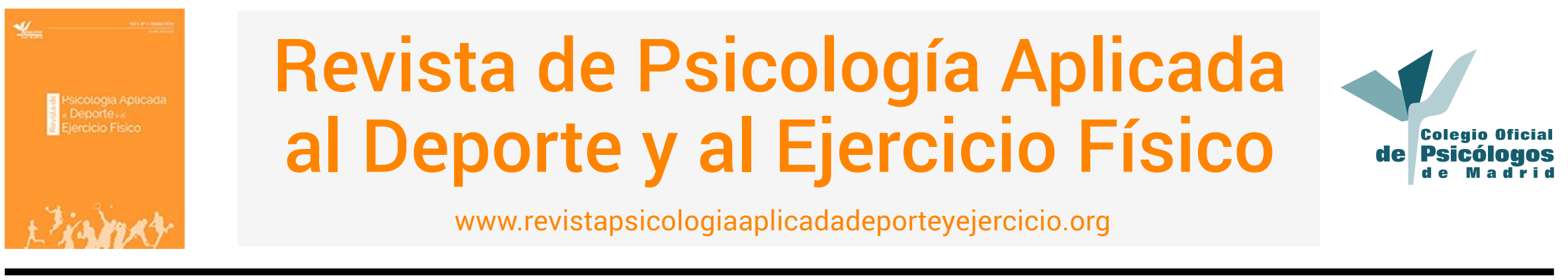

\title{
Entrenamiento psicológico mediante el coaching motivacional en alto rendimiento: una experiencia en marcha atlética
}

\author{
Julia Rodríguez Romeroa. Miryam Maestre Baidez ${ }^{\mathrm{b}}$ y Enrique Cantón Chirivella ${ }^{\mathrm{c}}$ \\ ${ }^{a}$ Colegio Oficial de Psicólogos de la Región de Murcia, España \\ ${ }^{\mathrm{b}}$ Universidad de Murcia, España \\ c Universidad de Valencia, España
}

\begin{abstract}
RESUMEN: En este artículo se presenta la intervención psicológica realizada con dos atletas de alto rendimiento de 19 y 23 años de edad cada uno. Se realizaron 10 sesiones de una duración media de 60-90 minutos cada una. Esta intervención se fundamenta en el coaching motivacional, en concreto, se utiliza el modelo de "La Jirafa de Cantón", donde el psicólogo genera un entorno facilitador en el que se resalta el papel activo del participante a la hora de analizar su proceso motivacional completo, sustentado en el conocimiento científico (Cantón, 2013). Respecto a la evaluación de su eficacia se utiliza un diseño A-B-A con los siguientes instrumentos: Escala de autoestima de Rosenberg (Rosenberg, 1989), Escala de Autoeficacia de Schwarzer y Baessler (1996) y Escala de Bienestar Psicológico de Ryff (Díaz et al., 2006). Los resultados muestran mejoras en las variables analizadas (bienestar psicológico, autoestima y autoeficacia), siendo la más notoria en Autoeficacia, coincidiendo este dato en ambos participantes. Se concluye que el coaching es una estrategia de intervención psicológica eficaz en modificación de la conducta en deportistas de alto nivel.
\end{abstract}

PALABRAS CLAVE: coaching deportivo; entrenamiento psicológico; bienestar psicológico; autoeficacia; autoestima.

\section{Psychological training through motivational coaching in high performance: an athletic running experience}

ABSTRACT: This article reports the psychological intervention carried out with two high-performance walkers aged 19 and 23 years. This consisted of 10 sessions of an average duration of 60-90 minutes. Intervention was based on motivational coaching. The "Cantón's Giraffe" model, where the psychologist generates a facilitating environment that emphasizes the active role of the participants in the complete motivational process, based on scientific knowledge, was specifically used (Cantón, 2013). To assess its effectiveness, an A-B-A design was used with the following instruments: Rosenberg Self-Esteem Scale (Rosenberg, 1989), Schwarzer and Baessler SelfEfficacy Scale (1996), and Ryff's Psychological Well-Being Scale (Díaz et al., 2006). The results show improvements in the analyzed variables (psychological well-being; self-efficacy; self esteem), most notably in self-efficacy in both participants. It is concluded that coaching is an effective psychological intervention strategy for modifying behavior in high-level athletes.

KEYWORDS: Sports Coaching, Psychological Training, Psychological Well-Being, Self-Efficacy, Self Esteem.

Treino psicológico através de coaching motivacional em alto rendimento: uma experiência em corrida atlética

RESUMO: Este artigo discute a intervenção psicológica realizada com dois atletas de alto rendimento com idade compreendida entre os 19 e os 23 anos. Foram realizadas 10 sessões com uma duração média de 60 a 90 minutos cada. Esta intervenção baseia-se no coaching motivacional, mais especificamente, no modelo "La Jirafa de Cantón", onde o psicólogo cria um ambiente propício no qual o papel ativo do participante é realçado quando analisa o seu processo motivacional completo, tendo por base o conhecimento científico (Cantón, 2013). Em relação à avaliação da sua eficácia é utilizado um desenho A-B-A com os seguintes instrumentos: escala de autoestima de

\footnotetext{
Julia Rodríguez Romero es psicóloga. Miriam Maestre Baidez es psicóloga y profesora en la Universidad de Murcia; Enrique Cantón Chirivella es psicólogo y professor de Psicología en la Universidad de Valencia.
} 
Rosenberg (Rosenberg, 1989), escala de autoeficácia de Schwarzer e Baessler (1996) e escala de bem-estar psicológico de Ryff (Diaz et al ., 2006). Os resultados apresentam melhorias nas variáveis analisadas (bem-estar psicológico; autoestima e autoeficácia), sendo a mais notória na Autoeficácia e sendo que esses dados coincidem em ambos os participantes. Conclui-se que o coaching é uma estratégia de intervenção psicológica eficaz na modificação do comportamento em atletas de alto nível.

PALAVRAS-CHAVE: coaching desportivo; treino psicológico; bem-estar psicológico; autoeficácia; autoestima.

Artículo recibido: 20/05/2018 | Artículo aceptado: 25/10/2018

En la Psicología del Deporte existe un creciente interés sobre el estudio del bienestar y la promoción de la salud psicológica de los deportistas, desde un punto de vista preventivo y del desarrollo, relacionado con la práctica de la actividad física y el deporte (Cantón, 2010; Liberal, Escudero, Cantallops, y Ponseti, 2014).

Dentro de las posibles líneas de intervención que integra la Psicología y que se focalizan en la perspectiva positiva del desarrollo de competencias y habilidades personales, el coaching se ha mostrado como una estrategia que ha obtenido resultados efectivos en la promoción y la mejora del bienestar y la salud general de las personas (Gorczynski, Morrow, e Irwin, 2008; Gordon-Bar, 2014). El coaching en el deporte es un proceso de entrenamiento-aprendizaje que consiste en activar el talento y los recursos del deportista para incrementar al máximo su rendimiento deportivo y personal, y eliminar los obstáculos internos a través del establecimiento de un plan de acción (cambio) con metas u objetivos. (Gutiérrez y Scheele, 2011a). El proceso de coaching está constituido por la escucha, interpretación y generación de preguntas por parte del coach, que sirve de guía al deportista para que este llegue a sus propias conclusiones gracias al entorno facilitador que se genera en este proceso (Buceta y Pérez-Llantada, 2009; Peris-Delcampo, 2012).

El coaching se define como una estrategia de intervención psicológica ya que produce cambios mantenidos en la cognición, la emoción y la conducta del cliente (García-Naveira, 2013). Representa un proceso de cambio que cubre el espacio entre "lo que uno es" y "lo que puede ser" y/o entre la situación actual (habitualmente no deseada por el cliente) y la situación ideal (deseada). Si se dan estas modificaciones se puede mejorar la promoción del bienestar y la salud psicológica (Cantón, 2014b; Gordon-Bar, 2014; Moore, Highstein, Tschannen-Moran y Silverio, 2010).

A modo orientativo, las etapas dentro de un proceso de coaching son (García-Naveira, 2011; Villa y Caperán, 2010): 1) generar el contexto y el vínculo, 2) establecer la situación actual, 3) establecer la situación ideal, 4) generar aprendizajes, 5) desarrollar un plan de acción y 6) pasar a la acción, interiorizar y consolidar.

El empleo del coaching ha ido creciendo en los últimos 30 años en diferentes áreas de desempeño de las personas como son la educación, la salud, la empresa o el deporte (García-Naveira, 2012; Valderrama, 2009). En concreto, en el contexto deportivo se ha ido extendiendo en el deporte de alto rendimiento, otros niveles de competición y en el deporte de ocio, salud y tiempo libre para la mejora del desempeño profesional y/o personal, el desarrollo del talento deportivo y la búsqueda de resultados (García-Naveira, 2011).

En general, se ha observado que la intervención en coaching produce cambios de comportamientos para la promoción de la salud de los individuos en áreas como las enfermedades crónicas (Hermens, op den Akker, Tabak, Wijsman, Vollenbroek, 2014), la obesidad (Newnham-Kanas, Irwin y Morrow, 2008; Zandvoort, Irwin y Morrow, 2009) o para dejar de fumar (Mantler, Irwin y Morrow, 2010), entre otras. Y también produce cambios en el bienestar de las personas como consecuencia del aumento de su autonomía, autoeficacia, autoestima, el desarrollo de aprendizajes, las expectativas positivas, el establecer y trabajar por unos objetivos propios, el esclarecer las acciones y estrategias de afrontamiento a realizar, y el refuerzo positivo por los avances o logros (Duijts, Kant, Van der Brant y Swaen, 2007; Gil-Monte, 2014; Wang, 2012).

En el contexto deportivo, García-Naveira (2016) y García-Naveira, García-Más, Ruiz y Cantón (2017) estudiaron la relación entre el coaching, el bienestar y la salud psicológica en deportistas adultos de rendimiento y jóvenes atletas de alto rendimiento. Los resultados indican que los deportistas poseen una buena percepción de bienestar y una mala salud psicológica, y que el grupo experimental, tras la aplicación del programa de coaching, mejora su percepción de salud psicológica y bienestar (en los casos que lo requiere). Estos cambios no se observaron en el grupo control. Los autores concluyen que si una intervención aporta mejoras en la vida de una persona se favorece su bienestar y su salud psicológica percibida.

Entre las variables psicológicas que intervienen en el rendimiento deportivo, y que se estudian en este trabajo, se encuentran la autoestima (Slutzky y Simpkins, 2009) y la capacidad de percibirse a sí mismo capaz de realizar determinadas tareas. Esta última variable es conocida como autoeficacia percibida y ha sido definida como "los juicios de cada persona sobre sus capacidades, en base a los cuales organizará y ejecutará sus actos de modo que le permitan alcanzar el 
rendimiento deseado" (Bandura, 1987, p. 416). Esta variable motivacional podría influir, en gran medida, en los resultados del deportista, constituyendo, por ello, un aspecto clave a trabajar por entrenadores, psicólogos del deporte y por los propios deportistas (Bandura, 1997; Godoy-Izquierdo, Vélez y Pradas, 2009; Ortega, Giménez y Olmedilla, 2008).

En el ámbito del rendimiento deportivo existen diversos estudios que demuestran la relación positiva existente entre el nivel de autoeficacia percibida y el rendimiento deportivo (García-Naveira, 2018; Feltz, Short y Sullivan, 2008; Nicholls, Polman y Levy, 2010). Además, autores como Besharat y Pourbohlool (2011) han demostrado que la autoeficacia tiene efectos moderadores de la influencia de la ansiedad competitiva sobre el rendimiento deportivo, sugiriendo que las expectativas de autoeficacia que presenta un deportista podrían tener efectos importantes en los patrones de pensamiento y comportamiento durante la competición (Barker, Jones, y Greenless, 2013; Feltz et al., 2008).

Con respecto a la autoestima, este constructo se considera como un componente o vertiente valorativa del autoconcepto (Marsh y Craven, 2006). Integraría, por tanto, componentes cognitivos y conductuales (Schneider, Dunton y Cooper, 2008). El componente cognitivo hace alusión al esquema global de cada individuo sobre sí mismo y el componente afectivo consiste en una autovaloración atribucional del logro por parte del propio sujeto (Mruk, 2006). La autoestima es una variable relevante respecto a las habilidades físicas en el ámbito del rendimiento deportivo (Fox, 2000; Jawaher, Dorsa, Ines, Imen y Abdelaziz, 2015; Molina, Chorot, Valiente, Sandín, 2014). Diversos estudios han relacionado la autoestima con la autoeficacia (Bandura, 1997), con el logro y el éxito (Mruk, 2006) y con la participación deportiva (Bowker, 2006).

La aplicación de modelos psicológicos desde el enfoque de coaching es un reto de especialistas en psicología y otros profesionales que buscan potenciar el rendimiento humano facilitando las condiciones para que el sujeto objeto de análisis (deportista) encuentre por sí mismo sus mejores soluciones para la consecución de objetivos (Peris-Delcampo, 2014). Fruto de esta necesidad y con motivo de crear una propuesta de trabajo motivacional aplicada y útil, se creó el modelo "La Jirafa de Cantón" (Cantón, 2013, 2014a) con características formales y conceptuales, basadas en la evidencia científica, sustentándose así en las teorías de la Motivación de Logro (McClelland, 1989), el modelo de Autoeficacia (Bandura, 1977, 1982, 1987; White 1959), la teoría de la Fuerza Motivacional o modelo de "Expectativas x Valor" (Atkinson, 1957, 1983; Tolman, 1955), y la Teoría de Metas (Deci y Ryan, 1985; Dweck, 1991; Ford, 1992; Locke, 1968), todos ellos como parte fundamentales de los aspectos bási- cos de las teorías motivacionales consolidadas en la ciencia psicológica (Palmero, Fernández-Abascal, Martínez y Chóliz, 2001; Reeve, 2010).

Un ejemplo de estudio que utiliza el mismo modelo es el de Peris-Delcampo, Expósito, y Cantón (2016). Este artículo presenta la intervención psicológica realizada con una mujer deportista de 19 años que presenta una discapacidad física y cuyo objetivo principal es el de acabar una travesía a nado en mar abierto. Para ayudarla en el logro de su meta, se realizan siete sesiones con una frecuencia de una vez a la semana tomando como punto de partida el modelo de coaching motivacional "La Jirafa de Cantón", donde el psicólogo genera un entorno facilitador para que el cliente descubra y encuentre sus propias soluciones. En líneas generales, los resultados muestran una mejora en las variables psicológicas de autoestima general, autoeficacia, percepción de control y motivación intrínseca.

Colás, Expósito, Peris-Delcampo y Cantón (2017) también realizaron una intervención a través del modelo de coaching "La Jirafa de Cantón" con una jugadora de fútbol sala de 18 años cuyo objetivo principal era llegar al fútbol sala profesional. Para ayudarla en el logro de su meta, se realizan nueve sesiones con una frecuencia de una vez a la semana y obtuvieron resultados eficaces declarando un efecto positivo en las variables psicológicas medidas, concretamente en la autoestima, algunas dimensiones de bienestar psicológico (relaciones positivas, dominio del entorno y propósito en la vida) y la percepción de autoeficacia general.

Partiendo de estas cuestiones, dentro de un proceso pre y pos intervención, los objetivos del presente trabajo son: conocer los componentes implicados en la motivación en este caso; detectar las fortalezas y carencias en los procesos motivacionales; fomentar la participación activa de la persona en su proceso de cambio y mejora mediante el compromiso en la realización de tareas; favorecer la reflexión sobre posibles estrategias para alcanzar la meta; y por último, evaluar la eficacia de la intervención aplicada en las variables autoestima, bienestar psicológico y autoeficacia, en las que se espera un incremento con respecto a la línea base (medida realizada antes de la intervención).

La hipótesis derivada de estos objetivos son que las puntuaciones en Autoestima, Autoeficacia General y Bienestar Psicológico de los deportistas se verán aumentadas tras la intervención en coaching.

\section{Participantes}

Los participantes son dos deportistas de alto rendimiento en marcha atlética, de 19 y 23 años de edad, con una 
experiencia deportiva en esta modalidad de 15 y 12 años respectivamente. Los dos atletas compiten a nivel internacional y entrenan 6 días a la semana con un total de 20 horas semanales.

La intervención se llevó a cabo por una psicóloga de 26 años con formación especializada en Psicología del Deporte (Máster en Psicología del Deporte y el Ejercicio, COP-CV) y 3 años de experiencia laboral. Previamente a la investigación, se realizó un entrenamiento en el modelo de "La Jirafa de Cantón" (Cantón, 2013) durante un total de 5 horas impartidas por Enrique Cantón Chirivella.

El consentimiento informado acerca de la participación voluntaria en la investigación y la confidencialidad de los datos lo firmaron ellos mismos ya que en el momento en el que se realizó el estudio ya eran mayores de edad.

\section{Instrumentos}

La metodología utilizada en este estudio fue pretest-postest con 3 fases: A) medida pre intervención; B) intervención y A) medida pos intervención, es decir consistió en un diseño A-B-A (Frías, Pascual y García, 1999). Las variables que se midieron fueron la autoestima, la autoeficacia y el bienestar psicológico. Para ello, se han utilizado los siguientes instrumentos:

Escala de Autoestima de Rosenberg (Rosenberg, 1989). Dicha escala tiene como principal objetivo evaluar la actitud personal de respeto y aceptación hacia sus propios valores (Rosenberg, 1965). Las características psicométricas de este instrumento han sido también estudiadas en el idioma español, obteniendo un alfa de Cronbach de .80 (Vázquez Morejón, Vázquez-Morejón Jiménez y Bellindo, 2013). Consta de 10 ítems de fácil comprensión (Abalde y Pino, 2016). El formato de respuesta es de tipo Likert con puntuaciones de 1 al 4 ( 1 = Muy en desacuerdo hasta 4 = Muy de acuerdo).

Escala de Autoeficacia de Schwarzer y Baessler (1996). Este instrumento tiene como principal objetivo medir la percepción estable de competencia para manejar de forma eficaz una gran variedad de situaciones estresantes. El cuestionario original consta de 10 ítems con una escala de respuesta tipo Likert de 4 puntos. Sin embargo, el utilizado en este estudio consta del mismo número de ítems (10) pero cambia el formato de respuesta, siendo en este caso una escala tipo Likert de 10 puntos donde se puntúa el grado de aplicabilidad del ítem a uno mismo (1 significa "en absoluto" y "10" significa "totalmente de acuerdo"). Esta versión fue revisada por Sanjuán, Pérez y Bermúdez (2000), la cual tiene un alfa de Cronbach de 87.

Escala de Pienestar Psicológico de Ryff (Díaz et al., 2006). Ryff (1989a, 1989b) sugirió un modelo multidimensional de bienestar psicológico compuesto por seis dimensiones: autoaceptación, relaciones positivas con otras personas, autonomía, dominio del entonro, propósito en la vida y crecimiento personal. Para medir estas dimensiones teóricas, Ryff desarrolló el instrumento conocido como "Escalas de Bienestar Psicológico" (Scales of Psychological Well-Being, SPWB) (Van Dierendonck, 2004). Consiste en un cuestionario de 29 ítems, con una escala Likert de 6 puntos (1 "completamente en desacuerdo, 2 "en desacuerdo", 3 "parcialmente de acuerdo", 4 "parcialmente de acuerdo", 5 "de acuerdo" y 6 "completamente de acuerdo"). La escala posee seis dimensiones: 1) Autoaceptación; 2) Relaciones positivas; 3) Autonomía; 4) Dominio del entorno; 5) Crecimiento personal, y 6) Propósito en la vida, en la que a mayor puntuación en cada una de ellas representa una mejor percepción de bienestar. En ocasiones también se ha considerado como una única dimensión sumando las puntuaciones de todos sus ítems, cuestión que ha sido adoptada en el presente trabajo. La escala utilizada en este estudio es la adaptación española de Díaz et al. (2006) que obtuvo un alfa de Cronbach entre .70 y .84 en función de sus componentes.

Además, se realizó una entrevista personal para conocer el seguimiento y la valoración a nivel personal del proceso de intervención. Las preguntas utilizadas en ambos casos fueron:

- ¿Crees que ha sido de utilidad lo trabajado en las sesiones correspondientes a la "Jirafa de Cantón?

- ¿Crees que de cara a esta nueva temporada todavía puedes beneficiarte de sus efectos?

- ¿Crees que las personas de tu círculo de confianza han notado esos efectos, siendo así, reflejado en las relaciones con estos?

\section{Procedimiento}

Se llevaron a cabo un total de 10 sesiones (una sesión a la semana) con cada deportista. Cada sesión, realizada en el centro deportivo donde entrenaban, en un despacho que reunía las condiciones necesarias para su uso (confidencialidad, sonido, temperatura e iluminación), tuvo una duración media de 60 minutos. El día de la sesión coincidía con un día de entrenamiento, previamente acordado entre las partes, 1.5 horas antes del mismo (16:00 horas).

El modelo utilizado en estas intervenciones se denomina "La Jirafa de Cantón" (Cantón, 2013). Se trata de una analogía que analiza el proceso motivacional completo, sustentado en el conocimiento científico que genera la investigación en Psicología.

En el modelo se identifican cuatro partes principales: 
1. Las patas: hace referencia al constructo de la motivación intrínseca puesto que se define como la base de todos los procesos motivacionales (Ortín y Jara, 2006). Su desarrollo se apoya en las diferentes formas de experiencia que facilitan la percepción de competencia, es decir, la autoconfianza (Revee, 2010): a) VEO: experiencia vicaria; b) OIGO: persuasión verbal y mi propio auto diálogo; c) SIENTO: interpretación de las señales psicofisiológicas y d) HAGO: experiencia directa.

2. El cuerpo: siendo las patas el punto de partida donde adquirimos la autoconfianza, en el cuerpo encontramos tres partes: a) PUEDO: percepción de autoeficacia; b) VALGO: valoración global de nosotros mismos, autoestima y c) SOY: el autoconcepto relacionado con ello.

3. El cuello: son las dos fuerzas que nos proyectan, esto es, CREO en mi capacidad para conseguirlo y VALORO mi objetivo. En otras palabras, expectativas de eficacia combinadas con la valoración incentiva del logro (fuerza motivacional).

4. La cabeza: se refleja el objetivo o logro buscado que puede analizarse siguiendo los criterios para un establecimiento de metas eficaz.
Para la aplicación del modelo, se distinguen tres sub-fases:

Primera sub-fase de evaluación general: el objetivo es determinar cómo se encuentra el participante en cada una de las partes. En este estudio se comienza por "la cabeza", para después dar un salto a la base y de ahí realizar la evaluación hacia arriba hasta llegar de nuevo a la meta.

Segunda sub-fase de sesiones intermedias: se trata de realizar un trabajo más específico potenciando las carencias encontradas en cada una de las partes, de cara a la consecución de la meta. Se distinguen dos tareas principales en cada sesión: repaso por parte del atleta y diálogo guiado por preguntas realizadas por el profesional.

Tercera sub-fase de cierre final: Repaso del estado general, refuerzo de cada aspecto importante y evaluación sobre la consecución de la meta.

A continuación, dentro de un proceso de pre y pos evaluación, se presenta la estructura del procedimiento de intervención en relación a la sesión, objetivo y desarrollo de la misma que se siguió con los participantes 1 y 2 del estudio (ver Tabla 1).

Tabla 1. Estructura del procedimiento de la intervención (Adaptación de Colás et al., 2017)

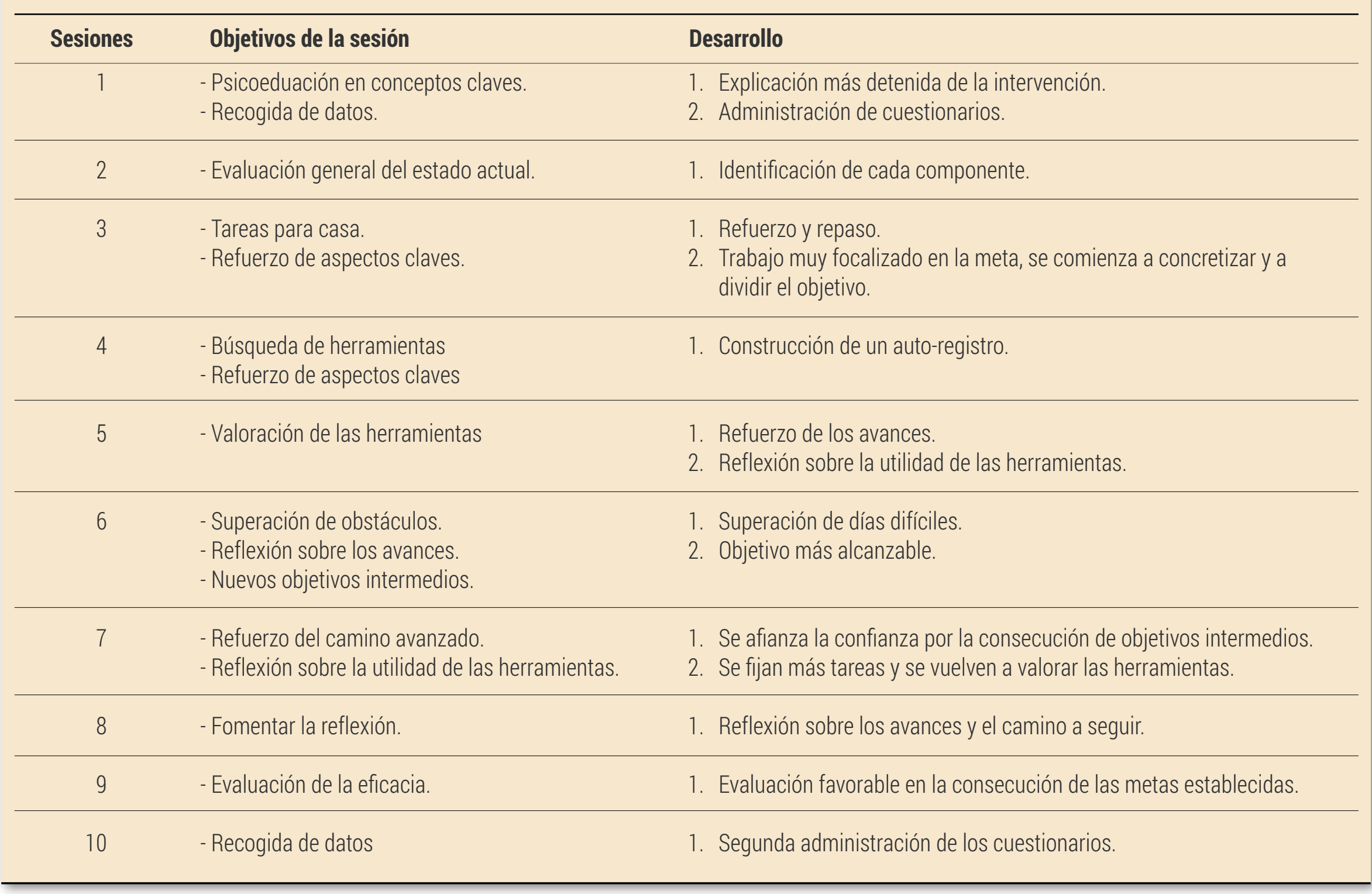




\section{Resultados}

En primer lugar, se presentan los objetivos establecidos por los deportistas, los cuales fueron trabajados durante las sesiones de coaching. El participante 1 se propuso una meta dirigida a la mejora de la autoconfianza y la autoestima tanto en competiciones como en entrenamientos. El participante 2 se propuso una meta dirigida a la consecución de ciertos hábitos y conductas de su vida cotidiana que creía necesarias para mejorar la calidad de sus relaciones familiares y deportivas.

En segundo lugar, se presentan los resultados de la evaluación (Autoestima, Bienestar Psicológico y Autoeficacia) en el tiempo 1 y 2 de cada deportista.

\section{Participante 1}

En la Gráfica 1 se presentan los datos correspondientes al participante 1. En lo referente al cuestionario de Autoestima, se obtuvo en la fase pre-test un total de 28 puntos y 27 en el pos-test, sin apenas variación entre ambas puntuaciones. Por lo que respecta a la escala de Autoeficacia, se obtuvo una puntuación de 72 puntos en el pre-test y 88 pos-test, por lo que existiría una diferencia de 16 puntos entre ambas. Por último, analizando las puntuaciones de la escala de Bienestar Psicológico (puntuación total), se encuentra una puntuación de 127 puntos en el pre-test y de 136 en el pos-test, lo que indica un aumento de 9 puntos entre ambas.

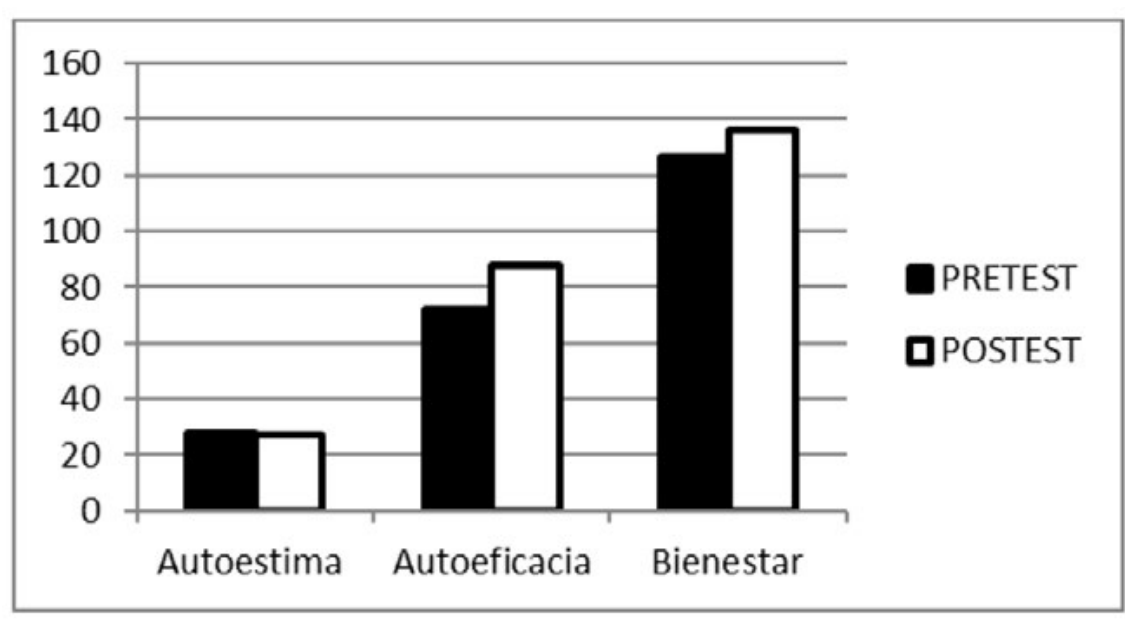

Gráfica 1. Puntuaciones en Autoestima, Autoeficacia General y Bienestar Psicológico pre y pos intervención (participante 1).

\section{Participante 2}

En la Gráfica 2 se encuentran los datos correspondientes al participante 2. En la escala Autoestima, se obtiene una puntuación total de 26 puntos en el pre-test y 27 en post-test, sin apenas variación entre ambas puntuaciones. Las puntuaciones de la escala de Autoeficacia se concretan en 60 puntos en el pre test y de 73 puntos en el post-test, por lo que refleja un aumento de 13 puntos entre ambas. Para finalizar, en la escala de Bienestar Psicológico, el participante obtiene una puntuación total de 128 en pre test y 125 en el post test, apenas con una variación de 3 puntos.

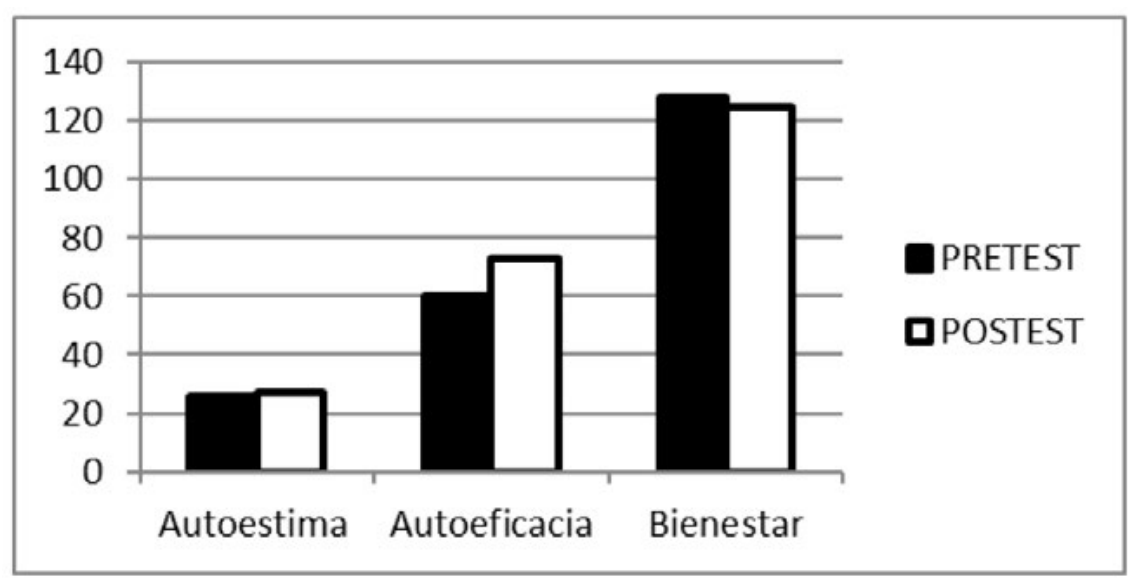

Gráfica 2. Puntuaciones en Autoestima, Autoeficacia General y Bienestar Psicológico pre y pos intervención (participante 2).

\section{Discusión}

Los datos obtenidos indican que, tras aplicar la intervención basada en el coaching en los dos marchadores de alto rendimiento, se confirma parcialmente la hipótesis planteada (las puntuaciones en Autoestima, Autoeficacia General y Bienestar Psicológico de los deportistas se verán aumentadas tras la intervención en coaching), ya que se observa un efecto positivo sobre la Autoeficacia (presentando un aumento de puntuaciones de 13 y 16 puntos respectivamente en cada deportista), aunque no se observan cambios representativos en las variables Autoestima (participantes 1 y 2) y Bienestar Psicológico, aunque en el participante 1 se percibe una cierta mejoría (tiempo 2).

Por ello, en la línea establecida por otros autores (Colás et al., 2017; García-Naveira, 2013, 2016; García-Naveira et al., 2017; Peris-Delcampo et al., 2016), el coaching se define como una estrategia de intervención psicológica en modificación de la conducta ya que produce cambios mantenidos en la cognición, la emoción y la conducta del cliente. En concreto, dentro del proceso de coaching, la "Jirafa de Cantón" se muestra en este caso eficaz en las variables psicológicas analizadas, con un total de 10 sesiones de 60-90 minutos cada una.

El aumento de la autoeficacia tras la aplicación del coaching es un resultado en el que se ha encontrado en otros trabajos (Colás et al., 2017; Peris-Delcampo et al., 2016). Esto indica que aumenta la percepción de competencia para manejar de forma eficaz ciertas situaciones estresantes, posiblemente estableciendo objetivos, aumentando sus recursos, mejorando la toma de decisiones, etc. Esta cuestión es importante ya que, como se ha observado, la autoeficacia 
se asocia positivamente con el rendimiento deportivo (García-Naveira, 2018; Feltz et al., 2008; Nicholls et al., 2010).

Además, al preguntar mediante entrevista personal a los atletas acerca de la utilidad de la intervención en un seguimiento a los tres meses, se obtiene información cualitativa de gran valor, ya que en la fase posterior al proceso de coaching ambos tienen la experiencia subjetiva de disponer de mayor número de herramientas para hacer frente a las situaciones. También, los agentes de socialización (entrenador, familiares y compañeros) que rodean a estos dos atletas comentaron de manera informal que perciben en ellos una mayor capacidad resolutiva con respecto a factores sensibles al rendimiento.

Respecto a la autoestima, no se observa variación en las puntuaciones tras la intervención. Estos datos van en contra de los obtenidos por Colás et al. (2017) y Peris-Delcampo et al. (2016). Por lo que parece, la intervención en coaching no ha afectado sobre la actitud personal de respeto y aceptación hacia sus propios valores en ninguno de los dos deportistas. En ambos casos, se podría definir como una autoestima media (puntuaciones entre 26-29 puntos; Rosenberg, 1989), siendo esta una variable a seguir estudiando en futuros trabajos.

Con respecto al bienestar psicológico se observan datos contrapuestos ya que uno de los deportistas presenta un efecto algo positivo en esta variable, con un aumento de 9 puntos posteriormente a la intervención, mientras que el otro deportista tiene un descenso de 3 puntos. En otros trabajos (Colás et al., 2017; Peris-Delcampo et al., 2016) si se obtiene una mejora del bienestar psicológico tras la intervención en coaching. Las investigaciones han demostrado que el bienestar psicológico de los deportistas suele ser positivo y con valores altos (García-Naveira, 2016). Por lo que es posible que en algunos casos el margen de mejora sea limitado según los objetivos establecidos de intervención en los que cada persona puede tener un "techo de bienestar psicológico", por lo que el coaching tendría mayor utilidad en personas con valores medios o bajos en esta cuestión (García-Naveira et al., 2017). Esta disparidad en los resultados también se podría explicar por la diferencia de experiencias (positivas o negativas) de cada uno de los deportistas cercanos a la evaluación, como resultados insatisfactorios en competiciones o entrenamientos, resultados académicos o relaciones interpersonales (Peris-Delcampo et al., 2016), que deberán ser registrados en futuros trabajos.

En relación a las metas trabajadas con cada deportista, como señalan García-Naveira (2016) y García-Naveira et al. (2017), estas pueden ser variadas y de diversos ámbitos (académico, laboral, familiar, interpersonal, deportivo, etc.), y no por ser deportistas se tiene que esperar necesariamente que las metas sean sobre el deporte. Lo importante es que el deportista decida qué quiere trabajar y que sea algo motivante para la persona.

Así, se debe considerar el ámbito del coaching como una estrategia de intervención psicológica que puede aportar mejoras en la vida de una persona, la consecución de metas y favorecer positivamente sobre ciertas variables psicológicas como la autoeficacia, entre otras (Colás et al., 2017; García-Naveira, 2016; García-Naveira et al., 2017; Martínez-Moreno, 2014; Peris Delcampo, 2015; Peris Delcampo et al., 2016).

Dentro de las limitaciones del estudio, como indican García-Naveira et al. (2017) y Colás et al., (2017), cabe destacar que para garantizar la no influencia de variables contaminadoras como el género, la edad, el número de sesiones y horas de aplicación del programa, o las que pueden generar el propio psicólogo que ha intervenido, hubiese sido necesario demostrar que estas variables no influyen sobre el resultado.

Para finalizar, se destaca la importancia de estos estudios en el ámbito profesional de la Psicología del Deporte, ya que en cuanto a su aplicabilidad ofrecen una herramienta de trabajo que permite identificar al psicólogo diferentes componentes de los procesos motivacionales, fundamentada desde la seriedad y la correcta aplicación de la Psicología (Cantón, 2013). Como perspectiva futura nos ofrece datos útiles para la elección de herramientas, para la mejora de la profesión y para las siguientes investigaciones (Cantón, 2016).

\section{Referencias}

Abalde, N. y Pino, R. (2016). Evaluación de la autoeficacia y de la autoestima en el rendimiento deportivo en Judo. Retos, 29(1), 109-113.

Atkinson, J. W. (1957). Motivational determinants of risk-taking behavior. Psychological Review, 64, 359-372. https://doi. org/10.1037/h0043445

Atkinson, J. W. (1983). Personality, motivation and action. Nueva York, Estados Unidos: Praeger.

Bandura, A. (1977). Social learning theory. Englewoods Cliffs, NJ, Estados Unidos: Prentice-Hall.

Bandura, A. (1982). Self-efficacy mechanism in human agency. American Psychologist, 37, 122-147. https://doi.org/10.1037/0003-066X.37.2.122

Bandura, A. (1987). Pensamiento y acción. Barcelona, España: Martínez Roca.

Bandura, A. (1997). Self-efficacy: The exercise of control. Nueva York, NJ, Estados Unidos: Freeman and Co. Publishers.

Barker, J. B., Jones, M. V. y Greenles, I. (2013). Using Hypnosis to Enhance Self-Efficacy in Sport Performers. Journal of Clinical Sport Psychology, 7, 228-247. https://doi.org/10.1123/ jcsp.7.3.228

Besharat, M. A. y Pourbohlool, S. (2011). Moderating Effects of Self- Confidence and Sport Self-Efficacy on the Relationship 
between Competitive Anxiety and Sport Performance. Psychology, 2, 760-765. https://doi.org/10.4236/psych.2011.27116

Boix, S., León-Zarceño, E. y Serrano-Rosa, M. A. (2016). Identificación con el ejercicio físico y autoeficacia: diferencias entre practicantes de pilates vs no practicantes. Revista Iberoamericana de Psicología del Ejercicio y el Deporte, 12(1), 99-106.

Bowker, A. (2006). The relationship between sports participation and selfesteem during early adolescence. Canadian Journal of Behavioural Science/Revue Canadienne des sciences du comportement, 38, 214-229. https://doi.org/10.1037/cjbs2006009

Buceta, J. M. y Pérez-Llantada, M. C. (2009). Características Generales del Coaching. Madrid, España: Dykinson.

Cantón, E. (2010). La psicología del deporte como profesión especializada. Papeles del Psicólogo, 31, 237-245.

Cantón, E. (2013). La "Jirafa de Cantón": un modelo de estrategia motivacional aplicado desde la perspectiva del coaching. Granada, España: Editorial CSV.

Cantón, E. (2014a). 'Cantón's Giraffe': A motivational strategy model applied from the perspective of coaching. The Coaching Psychologist, 10(1), 26-34.

Cantón, E. (2014b). ¿El coaching es Psicología? ¿Quién puede aplicar el coaching y en qué entornos? Informació Psicologica,107, 2-10.

Cantón, E. (2016). La especialidad profesional de la Psicología del Deporte. Revista de Psicología Aplicada al Deporte y al Ejercicio Físico, 1(2), 1-12. https://doi.org/10.5093/rpadef2016a2

Colás, J. M., Expósito, V., Peris-Delcampo, D. y Cantón, E. (2017). Intervención psicológica desde el coaching motivacional utilizando el modelo "La Jirafa de Cantón" en una jugadora de fútbol sala. Revista de Psicología Aplicada al Deporte y al Ejercicio Físico 2(10), 1-14. https://doi.org/10.5093/rpadef2017a12

Deci, E. L. y Ryan, R. M. (1985). The general causality orientations scale: Self-determination in personality. Journal of Research in Personality, 19, 109-134. https://doi.org/10.1016/00926566(85)90023-6

Díaz, D., Rodríguez-Carvajal, R., Blanco A., Moreno-Jiménez, B., Gallardo, I., Valle, C. y Dierendonck, D. (2006). Adaptación española de las escalas de bienestar psicológico de Ryff. Psicothema, $18572-577$.

Duijts, S., Kant, L., Van der Brant, P. y Swaen, G. (2007). The compatibility between characteristics of employees at risk for sickness absence and components of a preventive coaching intervention. International Journal of Evidence Based Coaching and Mentoring, 5(1), 19-29.

Dweck, C. S. (1991). Self-theories and goals: Their role in motivation, personality and developement. En R. A. Deinstbier (Ed.): Nebraska Symposium on Motivation 1990 (pp. 199-235). Lincoln, Estados Unidos: University of Nebraska Press.

Feltz, D., Short, S. y Sullivan, P. (2008). Self efficacy in sport: Research and strategies for working with athletes, teams and coaches. International Journal of Sports Science and Coaching, 3, 293-295.

Ford, M. E. (1992). Motivation humans: Goal, emotions and personal agency beliefs. Newbury Park, CA, Estados Unidos: Sage.

Fox, K. R. (2000). Self-esteem, self-perceptions and exercise. International Journal of Sport Psychology, 31, 228-240.

Frías, D., Pascual, J. y García, F. (1999). Principios del diseño de investigación. Valencia, España: Editorial CSV.
García-Naveira, A. (2011). Aproximación al empleo profesional del coaching en el deporte. Informació Psicológica, 101, 26- 39.

García-Naveira, A. (2012). Introducción al Coaching Deportivo. En A. García-Naveira (coord.): Liderazgo y Habilidades en Coaching Deportivo (pp. 13-28). Madrid, España: ABFútbol.

García-Naveira, A. (2013). Aplicación profesional del coaching en el deporte: un estudio de caso único. Cuadernos de Psicología del Deporte, 13(2), 101-112. https://doi.org/10.4321/S157884232013000200011

García-Naveira, A. (2016). Percepción del bienestar y de la salud psicológica, y la eficacia de un programa de intervención en coaching en deportistas de rendimiento. Revista Iberoamericana de Psicología del Ejercicio y el Deporte, 17(2), 211-219.

García-Naveira, A. (2018). Autoeficacia y rendimiento en jugadores de fútbol. Cuadernos de Psicología del Deporte, 18(2), 66-78.

García-Naveira, A., García-Mas, A., Ruiz-Barquín, R. y Cantón, E. (2017). Programa de intervención basada en el coaching en jóvenes deportistas de alto rendimiento, y su relación con la percepción de bienestar y salud psicológica. Revista de Psicología del Deporte, 26(2), 37-44.

Gil-Monte, P. (2014). Coaching cognitivo conductual: una herramienta para los psicólogos. Informació Psicológica, 107, 34-46.

Godoy-Izquierdo, D., Vélez, M. y Pradas, F. (2009). Nivel de dominio de las habilidades psicológicas en jóvenes jugadores de tenis de mesa, bádminton y fútbol. Revista de Psicología del Deporte, 18(1), 7-22.

Gorczynski, P., Morrow, D. e Irwin, J.D. (2008). The impact of coactive. Coaching on physically inactive 12 to 14 year olds in Ontario. International Journal of Evidence Based Coaching and Mentoring, 6(2), 13-26.

Gordon-Bar, S. (2014). How personal systems coaching increases self-efficacy and well-being for Israeli single mothers. International Journal of Evidence Based Coaching and Mentoring, 12(2), 59-73.

Gutiérrez, D. y Scheele, S. (2011a). Céntrate en el proceso y obtendrás resultados extraordinarios. En Conde, M. (Ed.). Actitud de triunfador (pp. 82-104). Pontevedra, España: Editorial Supérate.

Hermens, H., Op den Akker, H., Tabak, M., Wijsman, J. y Vollenbroek, M. (2014). Personalized coaching systems to support healthy behavior in people with chronic conditions. Journal of Electromyography and Kinesiology, 24, 815-826. https://doi.org/10.1016/j.jelekin.2014.10.003

Jawaher, M., Dorsa, T., Ines, F., Imen, B. y Abdelaziz, J. (2015). Athletic performance, self-esteem and temperamental profile: Which relationship? Tunisie Medicale, 93(3), 175-180.

Liberal, R., Escudero, J.T., Cantallops, J. y Ponseti, J. (2014). Impacto psicológico de las lesiones deportivas en relación al bienestar psicológico y la ansiedad asociada a deportes de competición. Revista de Psicología del Deporte, 23, 451-456.

Locke, E. A. (1968). Toward a theory of task motivation and incentives. Organizational Behavior and Human Performance, 3, 157189. https://doi.org/10.1016/0030-5073(68)90004-4

Mantler, T., Irwin, J. y Morrow, D. (2010). Assessing motivational interviewing through co-active life coaching tools as a smoking cessation intervention: a demonstration study. International Journal of Evidence Based Coaching and Mentoring, 8(2), 49-56.

Marsh, H. W. y Craven, R. (2006). Reciprocal effects of self-concept and performance from a multidimensional perspective: 
Beyond seductive pleasure and unidimensional perspectives. Perspectives on Psychological Science, 1, 133-163. https://doi. org/10.1111/j.1745-6916.2006.00010.x

Martínez-Moreno, A. (2014). Liderazgo y Coaching. Informació Psicológica, 107, 47-65.

McClelland, D. C. (1989). Estudio de la Motivación Humana. Madrid, España: Narcea.

Molina, J., Chorot, P., Valiente, R. M. y Sandín, B. (2014). Miedo a la evaluación negativa, autoestima y presión psicológica: Efectos sobre el rendimiento deportivo en adolescents. Cuadernos de Psicología del Deporte, 14(3), 57-66. https://doi.org/10.4321/ S1578-84232014000300007

Moore, M., Highstein, G., Tschannen-Moran, B. y Silverio, G. (2010). Coaching behavior change. En M. Moore y B. Tschannen-Moran (Eds.), Coaching psychology manual (pp. 33-51). Baltimore, MD, Estados Unidos: Lippincott Wiliams \&Wilkins.

Mruk, C. J. (2006). Self-Esteem: Research, Theory, and Practice: Toward a Positive Psychology of Self-Esteem ( $3^{\mathrm{a}}$ Ed.). New York, Estados Unidos: Springer.

Newnham-Kanas, C., Irwin, J. D. y Morrow, D. (2008). Co-active life coaching as a treatment for adults with obesity. International Journal of Evidence Based Coaching and Mentoring, 6(2), 1-12.

Nicholls, A. R., Polman, R. y Levy, A. R. (2010). Coping self-efficacy, pre-competitive anxiety, and subjective performance among athletes. European Journal of Sport Science, 10(2), 97-102.

Ortega, E., Giménez, J. M. y Olmedilla, A. (2008). Utilización del video para la mejora de la percepción subjetiva de la eficacia competitiva y del rendimiento en jugadores de baloncesto. Revista de Psicología del Deporte, 17(2), 279-290.

Ortín, F. y Jara, P. (2006). Estrategias para la motivación y el trabajo orientado por objetivos. En E. J. Garcés, A. Olmedilla y P. Jara. (Ed.), Psicología y Deporte (pp. 103.-123). Murcia, España: Diego Marín.

Palmero, F., Fernández-Abascal, E., Martínez, F. y Chóliz, M. (2001). Psicología de la Motivación y Emoción. México, México: McGrawHill.

Peris-Delcampo, D. (2012). Coaching en el deporte: claves para su correcta aplicación. Actas del $V$ Congreso Uruguayo de Psicología de la Actividad Física y el Deporte. 4-6 de octubre 2012.

Peris-Delcampo, D. (2014). El coaching: cómo podemos utilizarlo con éxito. Informació Psicologica, 107, 11-22.

Peris-Delcampo, D. (Julio, 2015). Trabajo psicológico a distancia con entrenadores y jugadores profesionales de fútbol. Comunicación presentada en el I Congreso Internacional sobre Psicología y Fútbol, Santiago de Compostela, España.

Peris-Delcampo, D., Expósito, V. y Cantón, E. (2016). Intervención psicológica en natación adaptada desde la perspectiva del coaching motivacional. Informació Psicológica, 112, 59-73.

Reeve, J. (2010). Motivación y emoción (5ª ed.). México, México: McGraw-Hill.

Ryff, C. (1989a). Beyond Ponce de Leon and life satisfaction: New directions in quest of successful aging. Internatio- nal Journal of Behavioral Development, 12, 35-55. https://doi. org/10.1177/016502548901200102

Ryff, C. (1989b). Happiness is everything, or is it? Explorations on the meaning of psychological well-being. Journal of Personality and Social Psychology, 57, 1069-1081. https://doi. org/10.1037/0022-3514.57.6.1069

Rosenberg, M. (1965). La autoestima del adolescente y la sociedad (traducción de 1973). Buenos Aires, Argentina: Paidós.

Rosenberg, M. (1989). Society and the adolescent self-image. Middletown, CT, Estados Unidos: Wesleyan University Press.

Sanjuán, P., Pérez, A. M. y Bermúdez, J. (2000). Escala de autoeficacia general: datos psicométricos de la adaptación para población española. Psicothema, 12(2) 509-513.

Schneider, M., Dunton, G. F. y Cooper, D. M. (2008). Physical activity and physical self-concept among sedentary adolescent females: An intervention study. Psychology of Sport and Exercise, 9(1), 1-14. https://doi.org/10.1016/i.psychsport.2007.01.003

Schwarzer, R. y Baessler, J. (1996). Evaluación de la autoeficacia: adaptación española de la escala de autoeficacia general. Ansiedad y Estrés, 2, 1-8.

Slutzky, C. B. y Simpkins, S. D. (2009). The link between children's sport participation and self-esteem: Exploring the mediating role of sport self-concept. Psychology of Sportand Exercise, 10(3), 381-389. https://doi.org/10.1016/i.psychsport.2008.09.006

Tolman, E. C. (1955). Principles of performance. Psychological Review, 62, 315-326. https://doi.org/10.1037/h0049079

Valderrama, B. (2009). Desarrollo de competencias de mentoring y coaching. Madrid, España: Pearson Educación.

Van Dierendonck, D. (2004) The construct validity of Ryff's Scale of Psychological well-being and its extension with spiritual well-being. Personality and Individual Differences, 36, 629-644. https://doi.org/10.1016/S0191-8869(03)00122-3

Vázquez-Morejón, A. J., Vázquez-Morejón Jiménez, R. y Bellindo, G. (2013). Fiabilidad y validez de la escala de Autoestima de Rosenberg (EAR) en pacientes con diagnóstico de psicosis. Apuntes de Psicología, 37, 37-43.

Villa, J. P. y Caperán, J. A. (2010). Manual de Coaching: cómo mejorar el rendimiento de las personas. Barcelona, España: profiteditorial.

Wang, Q. (2012). Coaching forlearning: exploring coaching psychology in enquiry-basedlearning and development of learningpower in secondaryeducation. Procedia - Social and Behavioral Sciences, 69(24), 177-186. https://doi.org/10.1016/j. sbspro. 2012.17.397

White, R. W. (1959). Motivation Reconsidered: The concept of competence. Psychological Review, 66, 297-333. https://doi. org/10.1037/h0040934

Zandvoort, M., Irwin, J. y Morrow, M. (2009). The impact of coactive life coaching on female university students with obesity. International Journal of Evidence Based Coaching and Mentoring, 7(1), 104-111. 